\title{
Blackbody radiation modified to enhance blue spectrum
}

\author{
Ching-Fuh Lin, * Cha-Hsin Chao, L. A. Wang, and Wei-Chung Cheng \\ Graduate Institute of Electro-Optical Engineering, National Taiwan University, Taipei 106, Taiwan
}

\begin{abstract}
Received September 21, 2004; revised manuscript received January 17, 2005; accepted January 21, 2005
Blackbody radiation is modified to enhance the blue spectrum with photonic boxes of $\sim 200 \mathrm{~nm}$. The modified blackbody radiation has two temperature-independent features. First, the enhanced blue light has the peak intensity pinched at $390 \mathrm{~nm}$ with an enhancement factor of over 5000 . This peak wavelength corresponds to the resonance wavelength of the largest-number boxes. Second, the spectral width is $90 \mathrm{~nm}$ and is governed by the variation of the box size. The physics can be easily explained by the significantly enhanced density of states at a certain spectrum as a result of photonic boxes. (C) 2005 Optical Society of America

OCIS codes: $230.6080,230.5750,030.5290$.
\end{abstract}

In 1879, Thomas Edison invented the light bulb that utilized blackbody radiation for illumination. ${ }^{1}$ This invention is still in common use today. However, the illumination efficiency remains low, because the visible spectrum $(400-700 \mathrm{~nm})$ of blackbody radiation occupies only $5 \%$ of the total radiation energy, even at the high temperature of $2200^{\circ} \mathrm{C}$. Increasing illumination efficiency is very attractive for the concern of energy consumption. Unfortunately, blackbody radiation is difficult to change. Recent advances in photonics have opened up the possibility of light manipulation by use of photonic crystals. ${ }^{2-8}$ Metallic photonic crystals thus have been shown to modify blackbody radiation for the enhancement of emission in IR spectrum. ${ }^{8,9}$ However, the physics of the enhancement is still not clear owing to the complication of photonic crystals. The modification of blackbody radiation for the enhancement of the visible spectrum also has not been shown because of the difficulty in their fabrication. Here we show another way to enhance blackbody radiation in the desired spectrum. Using photonic boxes with a size of $\sim 200 \mathrm{~nm}$, our experiment demonstrates that blackbody radiation has significantly enhanced light emission at around $400 \mathrm{~nm}$ with a narrow spectral width at a much lower temperature than a conventional situation. Because of the much simpler structure, its physics can be easily understood. Other visible spectra can also be enhanced by a simple increase in the size of photonic boxes.

The term "photonic box" is defined here as the metallic box that contains dielectric and has the dimension on the order of the wavelength of light. Assume that the box is a cube and the metal is a perfect conductor. According to the electromagnetic (EM) theory, the only EM wave that can possibly exist in the box has the wavelength given by $\lambda_{k l m}=2 n a /\left(k^{2}+l^{2}+m^{2}\right)^{1 / 2}$ (Ref. 10), where $n$ is the refractive index of the dielectric contained in the box and $a$ is the length of each side. $k, l$, and $m$ are either zero or positive integers, but at least two of them are nonzero. The allowable EM waves are called resonance modes, and the corresponding wavelengths are called resonance wavelengths.

Between the resonance wavelengths, there are no existing EM waves in the box. The forbidden spectrum, analogous to the terminology for photonic crystals, is called "band gap." Therefore photonic boxes have many "band gaps." In addition, there is a maximum wavelength beyond which no corresponding EM wave exists in the box. The maximum wavelength is given by $\lambda_{\text {cutoff }}=\sqrt{2} n a$, called the cutoff wavelength. Therefore photonic boxes have an extremely wide bandgap, which is almost infinite in principle. If the box has other shapes, for example, cylindrical shape or other geometric shapes, we can also analogously apply EM theory to calculate its resonance wavelengths. Their characteristics of enabling cutoff wavelength and suppressing a long-wavelength spectrum are similar to those of the cubic photonic box.

If a body consists of many photonic boxes that do not have the same size, the resonance wavelengths will form a band with its density of states (DOS) undulated by the size-distribution function. Then the bandgap between the resonance peaks shrinks and may disappear if the sizedistribution function is broad. However, the widest band gap that extends from the largest cutoff wavelength (corresponding to the largest box) to infinite wavelength should still exist. Figure 1 illustrates the DOS of a body containing many photonic boxes. The cases of a unique size and various sizes are shown. On the other hand, the photon statistics that favor long-wavelength spectrum ${ }^{11}$ will cause the DOS at the cutoff wavelength to dominate light emission.

In practice, the photonic boxes cannot exist by themselves; they are surrounded by metals. The emission of light thus consists of two parts. One is the EM wave in the photonic boxes. The other is from the surrounded metal that still follows the conventional blackbody radiation. Therefore we have the total radiation given by 


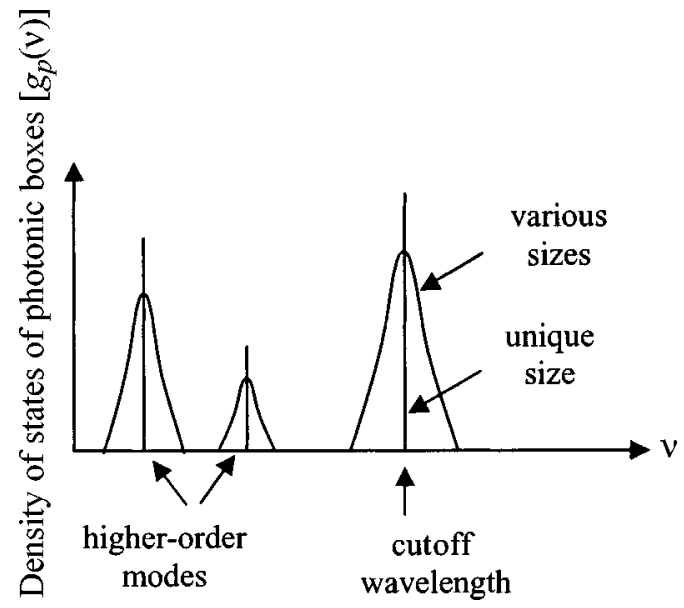

Fig. 1. Density of states of the photonic boxes. The unique box size will exhibit a delta function, and the various sizes of boxes will broaden the density of states.

$$
\begin{aligned}
\mu(\nu) \mathrm{d} \nu & =\left[V_{p} g_{p}(\nu)+V_{c} g_{c}(\nu)\right] \mathrm{d} \nu \times \bar{n}_{s} h \nu \\
& =\left[V_{p} g_{p}(\nu)+V_{c} \frac{8 \pi}{c^{3}} \nu^{2}\right] \frac{h \nu}{\exp \left(\frac{h \nu}{k T}\right)-1} \mathrm{~d} \nu,
\end{aligned}
$$

where $g_{p}(\nu)$ and $g_{c}(\nu)$ are the DOS functions (per unit volume) of the photonic boxes and the conventional blackbody, respectively; $V_{p}$ is the total volume of the photonic boxes, whereas $V_{c}$ is the total volume of the surrounded metal; and $\bar{n}_{S}$ is the photon statistics. The influence of the refractive index is implied in Eq. (1), because it will influence the field distribution and the cutoff wavelength. As a result, the refractive index will influence either the volume of the photonic box for achievement of the same effect at a certain wavelength or the density of states when the physical size of the boxes is fixed.

The term $V_{p} g_{p}(\nu)$ in Eq. (1) explains the blackbody radiation enhanced at the spectrum regulated by the DOS function. Let us define the enhancement factor $\eta$ by

$$
\eta=\left[V_{p} g_{p}(\nu)+V_{c} \frac{8 \pi}{c^{3}} \nu^{2}\right] / V_{c} \frac{8 \pi}{c^{3}} \nu^{2}=V_{p} g_{p}(\nu) / V_{c} \frac{8 \pi}{c^{3}} \nu^{2}+1 .
$$

If the enhancement factor is much larger than 1 , the modified blackbody radiation might exhibit temperatureindependent features characterized by the DOS function of the photonic boxes, $g_{p}(\nu)$.

The enhanced emission of blackbody radiation at the resonance wavelength by use of photonic boxes is verified by the following experiment. The resonance wavelengths are designed to be in the UV-to-blue light regime to show that the concept of photonic boxes can be easily applied to other wavelengths of visible light or IR radiation. The fabrication procedures are briefly described as follows. First, metal $\mathrm{Cr}-\mathrm{Au}-\mathrm{Pt}$ with the thicknesses of 10, 20, and 100 $\mathrm{nm}$, respectively, was sequentially evaporated on the $\mathrm{Si}$ substrate. Spin-on glass (SOG) was then deposited on top of the Pt metal. This SOG layer has a thickness of $220 \mathrm{~nm}$ and the refractive index of 1.4. Afterwards, photoresist (PR) was spun on the SOG. Immersion interference pho- tolithography (IIPL) ${ }^{12}$ with a 351-nm argon ion laser was applied twice to create two-dimensional periodic patterns on the PR. The patterned PR is supposed to consist of many periodically aligned $220 \mathrm{~nm} \times 220 \mathrm{~nm}$ squares, and the pitch of the pattern is $500 \mathrm{~nm}$. However, because it is not possible to accurately define $220-\mathrm{nm}$ features by use of UV lithography, the actual shapes are different. The patterned PR was used as the mask in the reactive-ionetching (RIE) process. During RIE, Ar and $\mathrm{CHF}_{3}$ gases were used to remove the SOG uncovered by PR. Before the removal of $\mathrm{PR}, \mathrm{Pt}$ was evaporated to fill up the area where the SOG was etched. The desired thickness of $\mathrm{Pt}$ is $220 \mathrm{~nm}$, same as the SOG. Then PR was rinsed off, so Pt on top of the PR was also removed. The processing was completed with depositing of a 10-nm Pt layer by use of evaporation. The top $\mathrm{Pt}$ layer is made only slightly thicker than its skin depth $(\sim 6 \mathrm{~nm})$ such that the resonant EM wave in the dielectric of the photonic boxes can leak out. The processing steps and the expected device structures after some processing steps are illustrated in Fig. 2. After the fabrication, many photonic boxes are formed. The interior of the photonic boxes is the dielectric of SOG, and the surrounding wall is Pt metal.

The photonic boxes were examined by use of atomicforce microscopy (AFM). Figure 3 shows the AFM image. The AFM is able to reflect each individual photonic box because the top surface is not flat, indicating that the evaporated Pt at step 5 (Fig. 2) does not have the exact same thickness as the SOG. However, because most boxes still have Pt surrounding the dielectric, the function of the photonic boxes is preserved. Because the IIPL method naturally results in periodic patterns, the fabricated boxes are aligned periodically, although it is not necessary to align them in this way for the enhanced spectrum at the resonance wavelength. The AFM image shows that the patterns have periods of 432 and $487 \mathrm{~nm}$, respectively, along two perpendicular directions. The shape is not rectangular, because the UV lithography cannot produce sharp corners, causing the resonance wavelength to be different from the theoretical design. However, the characteristics of photonic boxes are still preserved. Figure 3 also shows that the size exhibits variation, so there are various cutoff wavelengths, and the enhanced emission should have broadened spectrum.

The sample was placed on a graphite heating stage in a vacuum chamber with a quartz bell jar. The pressure was $2 \times 10^{-6}$ Torr. The emitted light was reflected by a mirror in the vacuum chamber and directed to the monochromator for spectrum measurement. A photomultiplier tube

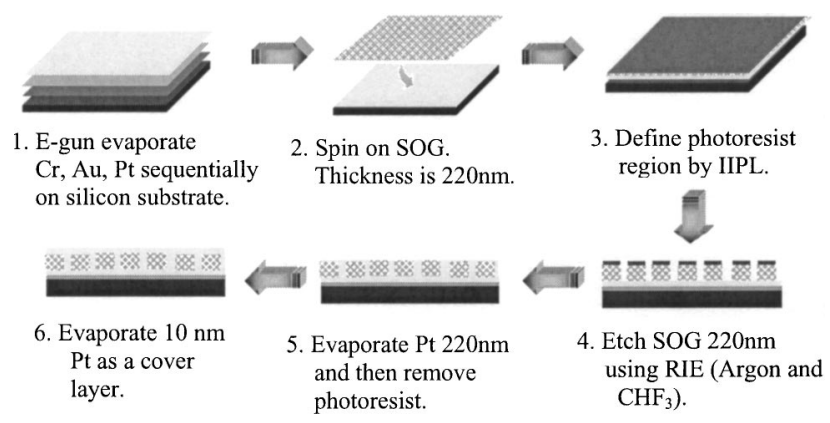

Fig. 2. Fabrication steps of the photonic boxes. 


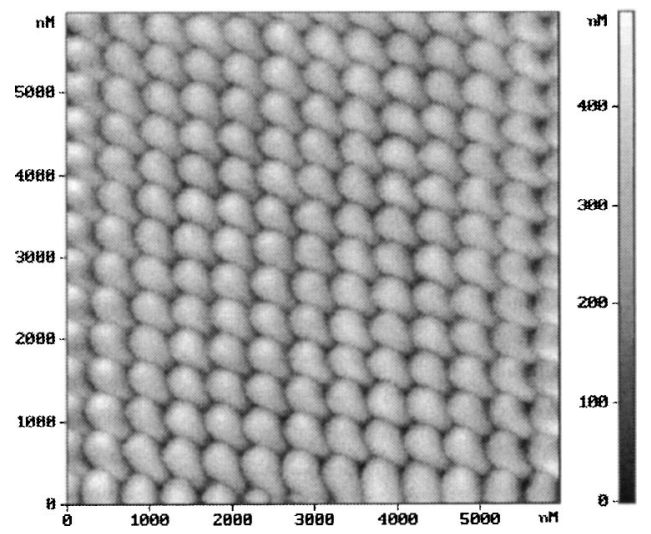

Fig. 3. AFM image of the fabricated photonic boxes.

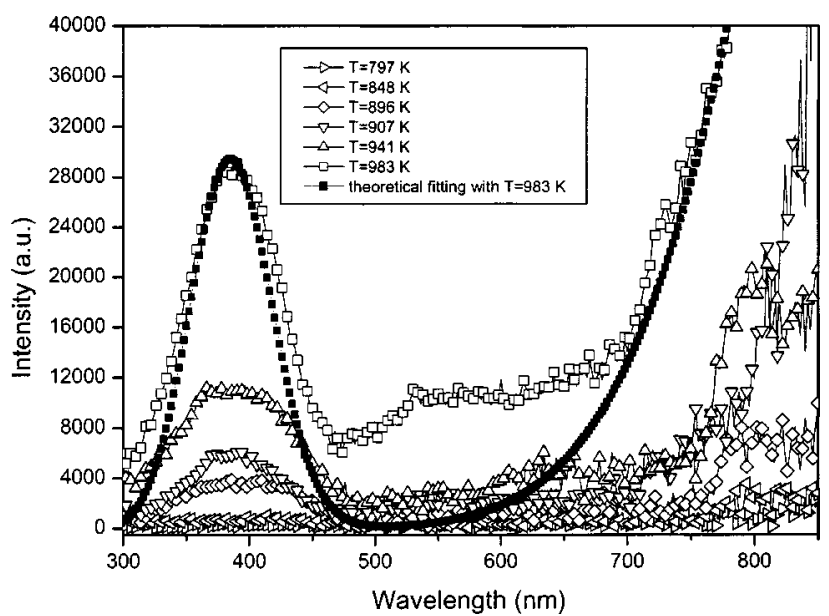

Fig. 4. Measured spectra of radiation from heated photonic boxes at different temperatures and a theoretical fitting with Eq. (1) at $T=983 \mathrm{~K}$.

(PMT; R928, Hamamatsu) was placed behind the monochromator for light detection. As a result, the measurement was limited to the spectral range of the PMT response. Spectra of radiation from the sample with photonic boxes at temperatures 797, 848, 896, 907, 941, and $983 \mathrm{~K}$ were measured and are shown in Fig. 4. Theoretical calculation at $983 \mathrm{~K}$ by use of Eq. (1) is also given in Fig. 4. The theoretical curve fits the experimental measurement pretty well. In the theoretical calculation, the DOS function of the photonic box $g_{p}(\nu)$ is approximated by a Gaussian function with a FWHM of $90 \mathrm{~nm}$. The metallic volume $V c$ is 4.16 times the volume of photonic boxes. This volume ratio is very close to the actual ratio of the fabricated sample.

The peak wavelength remains at approximately 390 $\mathrm{nm}$ at those temperatures, which is an important feature resulting from the DOS function of the photonic boxes, as described by Eq. (1). For temperatures not above $941 \mathrm{~K}$, the peak intensity at $390 \mathrm{~nm}$ is $\sim 6.5$ times larger than the background level at around $470 \mathrm{~nm}$. Assuming that the background level is the blackbody radiation with the DOS function $g_{p}(\nu)$ of the photonic boxes equal to zero, we have the following relation

$$
\begin{gathered}
{\left[V_{p} g_{p}\left(\nu_{1}\right)+V_{c} \frac{8 \pi}{c^{3}} \nu_{1}^{2}\right] \frac{h \nu_{1}}{\exp \left(\frac{h \nu_{1}}{k T}\right)-1} \cong 6.5} \\
\times V_{c} \frac{8 \pi}{c^{3}} h \nu_{2}^{2} \frac{h \nu_{2}}{\exp \left(\frac{h \nu_{2}}{k T}\right)-1}
\end{gathered}
$$

where $\nu_{1}$ and $\nu_{2}$ are the frequencies corresponding to the wavelengths of 390 and $470 \mathrm{~nm}$, respectively. Because

$$
V_{c} \frac{8 \pi}{c^{3}} \nu_{2}^{2} \frac{h \nu_{2}}{\exp \left(\frac{h \nu_{2}}{k T}\right)-1}>1000 \times V_{c} \frac{8 \pi}{c^{3}} \nu_{1}^{2} \frac{h \nu_{1}}{\exp \left(\frac{h \nu_{1}}{k T}\right)-1}
$$

for those temperatures, we have

$$
\begin{gathered}
{\left[V_{p} g_{p}\left(\nu_{1}\right)+V_{c} \frac{8 \pi}{c^{3}} \nu_{1}^{2}\right] \frac{h \nu_{1}}{\exp \left(\frac{h \nu_{1}}{k T}\right)-1}>6500} \\
\times V_{c} \frac{8 \pi}{c^{3}} \nu_{1}^{2} \frac{h \nu_{1}}{\exp \left(\frac{h \nu_{1}}{k T}\right)-1} .
\end{gathered}
$$

Therefore the enhancement factor $\eta$ at $390 \mathrm{~nm}$, defined by Eq. (2), is larger than 6500.

At the temperature of $983 \mathrm{~K}$, the peak intensity at 390 $\mathrm{nm}$ is $\sim 5$ times larger than the background level at 470 $\mathrm{nm}$, with an enhancement factor larger than 5000. The slightly reduced contrast is due to the fact that the photonic boxes are partially destroyed. The reason is twofold. First, because the top layer of $\mathrm{Pt}$ is only $\sim 10 \mathrm{~nm}$, this $\mathrm{Pt}$ film might break at high temperature as a result of the surface tension. Second, the substrate $\mathrm{Si}$ and metals $\mathrm{Cr}$, $\mathrm{Au}$, and Pt might form alloys at high temperature. Then the photonic-box structures are reshaped. The use of materials that sustain high temperature should improve the operational situation.

The spectral width is $90 \mathrm{~nm}$, which is about the same for all temperatures. This is another important feature characterized by the DOS function of the photonic boxes according to Eq. (1). As discussed above, it is due to the size variation of the photonic boxes, so the spectral width does not vary with the temperature.

The temperature-independent behaviors are different from those in the usual blackbody radiation but similar to the behaviors of metallic photonic crystals. However, the emission peak and the spectral width of the blackbody radiation of the metallic photonic crystals are due to the periodic nature..$^{8,9,13,14}$ In comparison, with photonic boxes, the blackbody radiation has the peak wavelength located at the resonance wavelength of the largest-number boxes and the spectral width governed by the size variation. If the photonic boxes have a single size, we expect that the emission should have a very narrow line. Our investiga- 
tion of photonic boxes gives a much clearer explanation on the physics of the blackbody radiation modified by photonic structures.

There is also an enhanced spectrum in the vicinity of $800 \mathrm{~nm}$. This is because some of the neighboring boxes have no Pt between them owing to the imperfect photolithography, causing two neighboring boxes to be connected together. As a result, one side of the box becomes twice as large as the design size, and the resonance wavelength is doubled. Although the number of those connected boxes is small, the favored long-wavelength spectrum of photon statistics ${ }^{11}$ leads to an emission at around $800 \mathrm{~nm}$ that is much more enhanced than that at around $400 \mathrm{~nm}$.

In conclusion, we demonstrate that photonic boxes with a size of about $200 \mathrm{~nm}$ are able to enhance the blue light of blackbody radiation. The enhanced blue light has a peak at $390 \mathrm{~nm}$ and a spectral width of $\sim 90 \mathrm{~nm}$, which are both temperature independent. The enhancement factor is more than 5000. The physics can be easily explained by the modified density of states as a result of photonic boxes. Blackbody radiation can also be modified to enhance other spectra by use of photonic boxes of different sizes or shapes.

This work is supported in part by the National Science Council, Taiwan.

*Also with Department of Electrical Engineering and Graduate Institute of Electronics Engineering.

\section{REFERENCES}

1. M. Pollard. Introduction to Solid-State Lighting (Wiley, New York, 2002), pp. 5-6.

2. E. Yablonovitch, T. J. Gmitter, and K. M. Leung, "Photonic band-structure: the face-centered-cubic case employing nonspherical atoms," Phys. Rev. Lett. 67, 2295-2298 (1991).

3. A. Genack and N. Garcia, "Observation of photon localization in a three-dimensional periodic array," Phys. Rev. Lett. 66, 2064-2067 (1991).

4. S. Y. Lin, E. Chow, V. Hietala, P. R. Villeneuve, and J. D. Joannopoulos, "Experimental demonstration of guiding and bending of electromagnetic waves in a photonic crystal," Science 282 274-276 (1998).

5. T. Baba, N. Fukaya, and J. Yonekura, "Observation of light propagation in photonic crystal optical waveguides with bends," Electron. Lett. 27, 654-655 (1999).

6. S. Noda, A. Chutinan, and M. Imada, "Trapping and emission of photons by a single defect in a photonic bandgap structure," Nature 407, 608-610 (2000).

7. M. U. Pralle, N. Moelders, M. P. McNeal, I. Puscasu, A. C. Greenwald, J. T. Daly, E. A. Johnson, T. George, D. S. Choi, I. El-Kady, and R. Biswas, "Photonic crystal enhanced narrow-band infrared emitters," Appl. Phys. Lett. 81, 4685-4687 (2002).

8. J. G. Fleming, S. Y. Lin, I. El-Kady, R. Biswas and K. M. Ho, "All-metallic three-dimensional photonic crystals with a large infrared bandgap," Nature 417, 52-55 (2002).

9. S. Y. Lin, J. Moreno, and J. G. Fleming, "Three-dimensional photonic-crystal emitter for thermal photovoltaic power generation," Appl. Phys. Lett. 83, 380-382 (2003).

10. D. K. Cheng, Field and Wave Electromagnetics, 2nd ed. (Addison-Wesley, Reading, Mass., 2000), pp. 584-586.

11. F. Reif, Fundamentals of Statistical and Thermal Physics (McGraw-Hill, New York, 1965).

12. W. C. Cheng, L. A. Wang, and C. Y Hsieh, "Phase masks fabricated by interferometric lithography for working in $248 \mathrm{~nm}$ wavelength," Microelectron. Eng. 67-68, 63-69 (2003).

13. S. Y. Lin, J. G. Fleming, E. Chow, and J. Bur, "Enhancement and suppression of thermal emission by a three-dimensional photonic crystal," Phys. Rev. B 62, R2243-R2246 (2000).

14. B. Temelkuran, H. Altug, and E. Ozbay, "Experimental investigation of layer-by-layer metallic photonic crystals," IEE Proc. Optoelectron. 145, 409-414 (1998). 